\title{
Oxygen saturation and breathing patterns in infancy. 1: Full term infants in the second month of life
}

\author{
V A Stebbens, C F Poets, J R Alexander, W A Arrowsmith, D P Southall
}

\begin{abstract}
Overnight 12 hour tape recordings were made of arterial oxygen saturation $\left(\mathrm{SaO}_{2}\right.$, pulse oximeter in the beat to beat mode) and abdominal wall breathing movement on 67 healthy, full term infants between the ages of 29 and 54 (median 39) days. The median baseline $\mathrm{SaO}_{2}$ during regular breathing was $99.8 \%$ (range $97 \cdot 0-100 \%)$. Fifty four infants (81\%) had shortlived episodes during which $\mathrm{SaO}_{2}$ fell to $80 \%$ or less (desaturation); the median rate was 0.9 desaturations/hour, and the median duration of each desaturation was 1.2 seconds. The 97th centile value for the duration of all episodes in which $\mathrm{SaO}_{2}$ fell to $\leqslant 80 \%$ was 4.0 seconds. The frequency of desaturations was significantly higher, and their duration significantly longer, when the breathing pattern was non-regular rather than regular. The percentage of apnoeic pauses $(\geqslant 4$ seconds in duration) followed by a desaturation was higher during non-regular than regular breathing; it was particularly high during periodic breathing.

A knowledge of normal variability of baseline measurements of oxygenation and of the relationship between oxygenation and breathing patterns in infants is essential to the use of pulse oximetry in clinical practice.
\end{abstract}

Pulse oximetry was introduced into clinical practice about 10 years ago. Since then many validation studies have been done with pulse oximeters made by different manufacturers. Some of these studies in infants have shown a close correlation with arterial oxygen saturation $\left(\mathrm{SaO}_{2}\right)$ measured by co-oximetry. ${ }^{1-4}$ It has been shown, however, that correlations might vary depending which pulse oximeter was used. ${ }^{5}$

In addition to its clinical use in monitoring oxygenation, pulse oximetry may be of value in cardiorespiratory physiology. ${ }^{6}$ It is a noninvasive technique, and can provide immediate information about sudden changes in arterial oxygenation.

In the clinical management of patients with cardiac or respiratory failure, or both, when accurate information about baseline measurements of oxygenation is particularly valuable, pulse oximeters are generally used in an 'averaging' mode. In such modes the displayed values are calculated as an average of the saturation measurements at each pulse during the preced- ing 2-15 seconds. For research and diagnostic purposes, however, it is possible to use some instruments without averaging several pulsesthat is, in a 'beat to beat' mode. This offers advantages: immediate information is available about any changes in oxygen saturation; these changes, combined with an assessment of the photoplethysmographic pulse waveforms, indicate when false saturation values are being caused by movement or other artefact; and, finally, artefactual signals are not averaged with non-artefactual signals.

Previous studies using pulse oximetry in infants have shown that sudden drops in oxygen saturation are relatively common in this age group, particularly in preterm infants and after short apnoeic pauses. ${ }^{7}$ To assess their clinical importance, reference data about patterns of oxygenation must be available. Baseline oxygenation and short lived episodes of hypoxaemia have therefore been documented from overnight 12 hour multichannel physiological recordings on a sample of 67 healthy infants in the second month of life. The relationship of these hypoxaemic episodes to breathing patterns and apnoeic pauses was determined.

\section{Patients and methods}

Using a systematic sampling procedure, 69 infants were recruited immediately after birth from all those born in one hospital who had a gestational age of at least 37 weeks and seemed healthy on medical examination in the nursery and subsequently. The study was performed at a median age of 39 days (range 29-54, interquartile range (IQR) 35-43) in the infants' homes. Recordings were made when the infants were in good health (that is, free of major symptoms from the parents' report and the researcher's observation) and not taking any drugs. Eight infants (12\%) had minor problems at the time of recording (snuffly nose $(n=2)$, thrush $(n=2)$, colic $(n=2)$, eye infection $(n=1)$, and eczema $(n=1)$ ). Fully informed consent was obtained from the parents of all infants recruited.

Overnight multichannel physiological recordings started between 1830 and 2200 hours (median 2052) and lasted for a mean (SD) of $11.5(1 \cdot 1)$ hours. Recordings were made of arterial oxygen saturation and each photoplethysmographic pulse waveform from a pulse oximeter (Nellcor N100 with new software 
equivalent to N200 and specially modified to provide beat to beat data). The oximeter sensor was taped around the lateral edge of the infant's foot. In addition, abdominal breathing movements from a volume expansion sensor (Graseby Dynamics, in $73 \%$ ) or respiratory inductance plethysmography system (Studley Data Systems, in $27 \%$ ) were recorded. The volume expansion capsule was taped with concentric strips of adhesive tape (3M Micropore) midway between the umbilicus and xiphisternum. Inductance plethysmography was obtained using a purpose made vest (PK Morgan).

Data were recorded onto a four channel FM tape recorder (Racal, frequency response DC to $313 \mathrm{~Hz}$, signal:noise ratio $47 \mathrm{~dB} /$ channel) at a tape speed of 15/16 inches/second. Oxygen saturation recordings were calibrated to markers of 0,50 , and $100 \%$ generated from the oximeter. The recordings were printed onto graph paper by an ink jet recorder at $3.2 \mathrm{~mm} /$ second. Two recordings (3\%) had poor quality oxygen saturation signals throughout and were removed from the study. Analysis of the remaining 67 recordings was done manually by two experienced workers according to the following criteria:

- Regular and non-regular breathing patterns-A regular breathing pattern was signified by episodes of at least one minute in duration during which the abdominal wall movement waveform was steady in rate and amplitude. ${ }^{8}$ All periods of the recording not conforming to the above definition, or periods in which the steady pattern was disrupted by body movements, frequent sighs, or apnoeic pauses were classified as non-regular breathing patterns. Episodes of periodic apnoea were included in non-regular breathing patterns. The classification of regular breathing has previously been shown to be reproducible. $^{8}$

- Pauses in breathing movements-These were classified as apnoeic if they lasted for $\mathbf{4}$ seconds or more measured from the end of the expiratory movement waveform to the onset of the inspiratory movement waveform (fig 1 ). Pauses were classified according to their duration (4.0$7 \cdot 9,8 \cdot 0-11 \cdot 9$, and $\geqslant 12$ seconds). Episodes in which there were three or more successive apnoeic pauses, each separated by less than 20 breaths, were classified as periodic apnoea. ${ }^{8}$

- Baseline oxygen saturation and episodic desaturation-Oxygen saturation measurements were made only after an assessment of the accompanying photoplethysmographic pulse waveforms. Any period in which the quality of this signal was unsatisfactory was excluded from the analysis (fig 1). The remaining oxygenation signal was measured in two ways. Baseline $\mathrm{SaO}_{2}$ was calculated for each episode of regular breathing by measuring the $\mathrm{SaO}_{2}$ values at the end inspiration for each of five successive breaths at the centre of each episode of regular breathing. These five breaths had to be at least 10 seconds away from sighs or apnoeic pauses.

During both regular and non-regular breathing patterns the duration of each episode in which $\mathrm{SaO}_{2}$ fell to $80 \%$ or below was measured (fig 1). The minimum duration measured was 0.3 seconds. These desaturations were classified

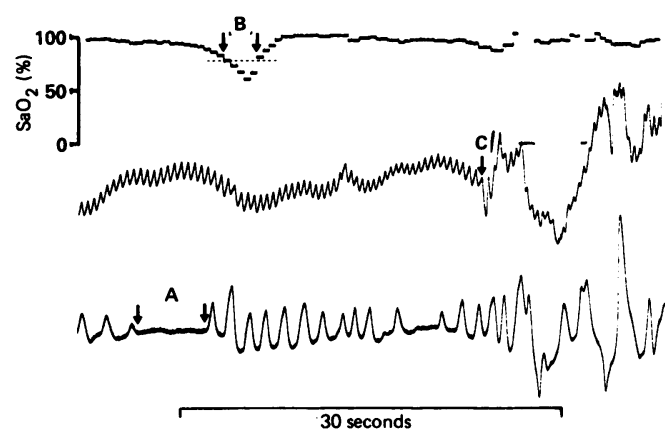

Figure 1 Section of a recording on an infant studied at the age of 35 days. The uppermost signal is oxygen saturation and the middle signal the plethysmographic pulse waveforms from which the saturation values were derived; both signals were generated from the pulse oximeter in the beat to beat mode. The lowest signal is abdominal wall breathing movements, showing a non-regular breathing pattern. At point $A$ there is an apnoeic pause with a duration of $5 \cdot 3$ seconds, which is associated with a period of desaturation $\leqslant 80 \%$ lasting $2 \cdot 5$ seconds $(B)$. The pulse signal can be analysed until point $C$, at which time body movement (note the increased volume of breathing movement) disrupts the waveform, and the subsequent saturation signal shows unreadable values.

as related to apnoeic pauses if the beginning of an apnoeic pause and of a subsequent desaturation were separated by a period of $2 \cdot 0-12 \cdot 0$ seconds. This time was defined after measurement of the total response time of the pulse oximeter in the beat to beat mode ${ }^{6}$ and examination of pilot data.

Rates of desaturation/hour of artefact free signal and the mean durations of these episodes were calculated for each recording. The significance of the differences was assessed by the Wilcoxon signed rank test, and correlations were tested by the Spearman rank correlation coefficient. Results are expressed as median (range) for individual recordings.

\section{Results}

All measurements of oxygenation were correlated with postnatal age at the time of recording. Coefficients for these correlations were all close to zero, and there was thus no evidence that age had an influence on the results within the range studied. The data are therefore presented without reference to age.

The median duration of regular breathing pattern was 3.0 hours (range $1 \cdot 1-5 \cdot 8$ ) and that of non-regular breathing pattern $8 \cdot 8$ hours $(6 \cdot 0$ $10 \cdot 3)$. The median duration of artefact free $\mathrm{SaO}_{2}$ signal was 2.9 hours $(1 \cdot 1-5 \cdot 4)$ during regular breathing pattern and $4 \cdot 6$ hours $(2 \cdot 3$ 6.9) during non-regular breathing pattern.

During the periods in which there was an artefact free $\mathrm{SaO}_{2}$ signal, all recordings showed apnoeic pauses, with a median value of $6 \cdot 8(0.4$ $72 \cdot 0)$ pauses/hour. Thirty three infants $(49 \%)$ had episodes of periodic apnoea. The median total duration of periodic apnoea in these infants was $0.3(0.1-14.5)$ minutes/hour.

The median baseline $\mathrm{SaO}_{2}$ was $99 \cdot 8 \%$ (range $97 \cdot 0-100 \%)$. Analysis of variability in baseline $\mathrm{SaO}_{2}$ measurements within individual recordings showed a median $\mathrm{SD}$ of $0.2 \%$ (range 0 $1 \cdot 4 \%)$. There was therefore low variability in 
baseline $\mathrm{SaO}_{2}$ among different episodes of regular breathing in the same recording.

Of 67 recordings, $54(81 \%)$ contained episodes in which the $\mathrm{SaO}_{2}$ fell to $80 \%$ or below, with a median of 0.9 episodes/hour (range $0-15 \cdot 1$ ) for all recordings. The median of the mean duration of these episodes for individual recordings was $1 \cdot 2$ seconds $(0 \cdot 3-2 \cdot 2)$. The median for the longest individual desaturation in each recording was $2 \cdot 4$ seconds $(0 \cdot 3-8 \cdot 6)$. Looking at all the desaturations, $97 \%$ lasted less than $4 \cdot 0$ seconds (only 24 desaturations in 11 recordings lasting for 4.0 seconds or longer).

The median number of desaturations/hour was $0 \cdot 0(0 \cdot 0-1 \cdot 7)$ during regular breathing compared with $1.5(0-23 \cdot 1)$ during non-regular breathing) $(p<0.001)$ (fig 2). The median of the mean durations of desaturations was 0.8 seconds $(0 \cdot 3-1 \cdot 6)$ during regular breathing compared with $1 \cdot 1$ seconds $(0 \cdot 3-2 \cdot 9)$ during non-regular breathing $(\mathrm{p}<0.05)$. During regular breathing $98 \%$ of desaturations were associated with an apnoeic pause; during non-regular breathing this proportion was $65 \%(\mathrm{p}<0.01)$.

The proportion of apnoeic pauses followed by a desaturation (table) varied with the duration of pause and the breathing pattern. The proportion was lowest during regular breathing (regardless of duration) and highest during periodic apnoea, particularly in association with long pauses. The differences were significant when pauses of $<8.0$ seconds were compared with pauses of $8 \cdot 0-11 \cdot 9$ seconds in duration during periodic apnoea $(p<0.05)$, and when isolated short pauses during regular compared with non-regular breathing were compared $(p<0.001)$. Pauses of $\geqslant 12.0$ seconds were so infrequent (10 in eight infants) that they did not

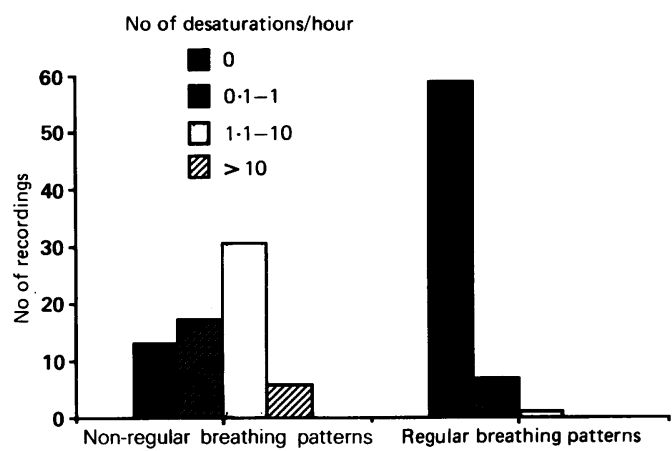

Figure 2 Numbers of recordings, subdivided into number of desaturations/hour of artefact free signals, for non-regular and regular breathing patterns.

Percentages of apnoeic pauses that were followed by desaturations. Medians (ranges) for differing pause durations during different breathing patterns. The number of recordings containing such pauses is given in parentheses below the results for each cell

\begin{tabular}{|c|c|c|c|}
\hline \multirow{3}{*}{$\begin{array}{l}\text { Duration of } \\
\text { apnoeic pause } \\
\text { (seconds) }\end{array}$} & \multicolumn{3}{|c|}{ Breathing pattern } \\
\hline & \multirow[t]{2}{*}{ Regular } & \multicolumn{2}{|l|}{ Non-regular } \\
\hline & & $\begin{array}{l}\text { Isolated } \\
\text { pauses }\end{array}$ & $\begin{array}{l}\text { Pauses in } \\
\text { periodic } \\
\text { apnoea }\end{array}$ \\
\hline $4 \cdot 0-7 \cdot 9$ & $\begin{array}{cc}0 & (0-46) \\
(n=64)\end{array}$ & $\begin{array}{c}9 \cdot 1(0-67) \\
(n=67)\end{array}$ & $\begin{array}{c}5 \cdot 9(0-67) \\
(n=33)\end{array}$ \\
\hline $8 \cdot 0-11 \cdot 9$ & $\begin{array}{c}0(0-50) \\
(n=24)\end{array}$ & $\begin{array}{r}0(0-100) \\
(n=34)\end{array}$ & $\begin{array}{c}36 \cdot 8(0-100) \\
(n=14)\end{array}$ \\
\hline
\end{tabular}

provide adequate information for analysis in any of the breathing/pause pattern categories.

To assess whether the infants with more pauses also had a higher percentage of pauses associated with desaturation we calculated the Spearman correlation coefficient for these two variables, but it was poor $(r=0 \cdot 23)$.

\section{Discussion}

We have studied reference data for variables concerning breathing movements patterns and oxygenation in full term infants at about 6 weeks of age. Interestingly, there were no obvious developmental changes in oxygenation over the age range included in the study. Analysis of similar recordings in a subgroup of infants studied sequentially between the ages of 6 weeks and 1 year showed no major changes in either baseline $\mathrm{SaO}_{2}$ or short lived falls in $\mathrm{SaO}_{2}$ during the period analysed (unpublished data). Thus arterial oxygenation seems to be relatively stable beyond the neonatal period.

The distribution of regular and non-regular breathing patterns has been described by Richards et al. ${ }^{8}$ The present results are similar to those of that study, in which 24 hour recordings of breathing movements and electrocardiograms in infants of comparable age were analysed. Assuming that a regular breathing pattern is highly correlated with a state of quiet sleep, ${ }^{9}$ our finding of three hours of regular breathing during an overnight recording is comparable with figures given in other studies for the duration of quiet sleep in this age group. ${ }^{1011}$ One limitation of this method is that both awake and active sleep periods are included in non-regular breathing. Information about differences in oxygenation and respiratory patterns between these states is therefore not available from this analysis.

Comparison of the frequency of apnoeic pauses with data from other studies is difficult, because there is no common definition of apnoeic pauses. ${ }^{12}$ Our results, however, are similar to those of Richards et al who included all apnoeic pauses of $\geqslant 3.6$ seconds in duration. ${ }^{8}$

The baseline oxygen saturation measurement made during regular breathing was high in all infants, with little variability between different measurement points in the same recording. Our values are higher than those given by Mok et al who used the same oximeter. ${ }^{13}$ They did not assess the quality of the photoplethysmographic waveforms, however, and so artefactually depressed values could have been incorporated in their results. In addition, their measurements were calculated as an average of all $\mathrm{SaO}_{2}$ values occurring at a fixed sampling interval, so they potentially included desaturations in their analysis and failed to distinguish between breathing patterns.

Desaturations were present in most infants, but they were usually short, $97 \%$ being less than 4 seconds long. During regular breathing almost all desaturations followed an apnoeic pause. During non-regular breathing a third of the desaturations were not preceded by apnoeic pauses of 4.0 seconds or more in duration. Some of these latter desaturations may have reflected 
a response to pauses shorter than 4 seconds; others may have been accompanied by continued abdominal wall movements, with or without continued airflow. ${ }^{7}$ Recordings that include respiratory airflow would be required to investigate these phenomena fully.

The proportion of apnoeic pauses followed by a desaturation may be an indicator of the oxygen stores in the lung, the oxygen consumption, and of the matching of ventilation to perfusion. Interestingly, less than half the recordings that showed apnoeic pauses during regular breathing showed any of these pauses followed by a desaturation to $80 \%$ or less. The proportion of short pauses associated with desaturations increased significantly during non-regular breathing. Active sleep is included in non-regular breathing, and this increase between regular and non-regular breathing patterns might, at least in part, be explained by an increase in oxygen consumption, and possibly a reduction in intrapulmonary oxygen stores, during active sleep..$^{14} 15$

During periodic apnoea, over half of the 14 recordings showing longer pauses $(8.0$ to 11.9 seconds) had more than one third of such pauses followed by a desaturation. Similar findings about the effect of periodic breathing on oxygenation were reported by Mok et al who, by using methods that absorbed episodic desaturation values into their average values of saturation, found a mean decrease of $26 \%$ from baseline measurements during periodic breathing. ${ }^{13}$ As only a relatively small number of infants in our study had apnoeic pauses of 8 seconds or longer, the apparently stronger influence of periodic breathing on oxygenation remains a preliminary result.

Reference data about episodes of desaturation in infants have not previously been published. In this study we used a pulse oximeter that has been validated against arterial line measurements in infants. ${ }^{3}$ We also used the oximeter in the beat to beat mode. Most infants, despite a high baseline measurement of $\mathrm{SaO}_{2}$, showed drops in oxygenation to levels below a threshold which has been previously defined as indicating an 'abnormal' reaction to an apnoeic pause. ${ }^{1617}$ Most of the recorded episodes, however, were brief ( $<4$ seconds) and would not, therefore, have been observed using a pulse oximeter that averages $\mathrm{SaO}_{2}$ values over several seconds. In the beat to beat mode, pulse oximeters can sense short lived falls in $\mathrm{SaO}_{2}$ that are not seen with the averaging modes used normally.

Because the short lived falls in $\mathrm{SaO}_{2}$ that were identified by this pulse oximeter occurred in healthy infants they must be regarded as normal. Variations in either baseline oxygenation measurements, or in the duration or frequency of episodes of desaturation beyond the range given here, however, may indicate a clinically relevant disturbance of oxygenation.

It might be argued that the short lived episodes of desaturation identified by this pulse oximeter in the beat to beat mode are artefactual, but their consistent relationship to apnoeic pauses, and previous observations that similar but more severe desaturations can occur during cyanotic episodes that have been documented as 'real' by arterial blood gas analysis, ${ }^{18}$ suggest that they do reflect true episodes of hypoxaemia. Moreover, similar short lived desaturations have been reported by other workers, not only using pulse oximetry, ${ }^{13}$ but also ear oximetry.

Recordings of breathing movements and electrocardiograms (pneumograms) are widely used to study respiratory physiology in infants. The frequency of apnoeic pauses and the quantity of periodic breathing have been used to indicate the need for home monitoring ${ }^{20}$ or treatment with methylxanthines. ${ }^{21}$ From a physiological viewpoint, however, hypoxaemia seems to be a better indicator of the adequacy of ventilation and ventilation/perfusion matching. ${ }^{18}$ Certainly these present results indicate that any recording of breathing movements without an indicator of oxygenation will produce information that is difficult to interpret. Firstly, there was poor correlation between the frequency of apnoeic pauses and the percentage of those pauses accompanied by desaturation; secondly, about a third of the desaturation episodes were not related to apnoeic pauses; and finally, the percentage of apnoeic pauses accompanied by episodes of desaturation, particularly during periodic apnoea, varied widely among individual infants.

In conclusion, our results indicate the importance of including oxygenation in studies of respiratory physiology, whether these be on healthy subjects or patients with symptoms. Although an outlying result in either the baseline oxygen saturation value or the frequency of recurrent short lived falls in $\mathrm{SaO}_{2}$ may be helpful in assessing the severity of illness in infants with symptoms, for instance during a respiratory tract infection, the prognostic value of outlying results in infants who are symptom free remains unknown. Further prospective studies are needed to answer this potentially important question.

We thank the workers who gave careful technical effort to the study: Mary Gray, who did the recordings, Pauline Mills and Jackie Kelly who made the measurements, and Linda Alexander and Vivienne Taylor who carried out basic analyses. Thanks also to the families in Doncaster who allowed us to use their time and homes, and made the study possible. Technical advice was and by DrW New, and computing facilities were donated by Hewlett by Dr W New, and computing facilities were donated by Hewlett Packard Ltd and Ashton-Tate Ltd. Mrs Stebbens was funded by the Nuffield Foundation, Dr Poets by the Deutsche Forschungsgemeinschaft, Bonn, Germany, and Dr Southall by the National Heart and Chest Hospitals, and Nellcor Inc. The study was supdon, and by the Cot Death Fund at Doncaster Royal Infirmary.

1 Hay WW, Brockway JM, Eyzaguirre M. Neonatal pulse oximetry: accuracy and reliability. Pediatrics 1989;83: 717-22.

2 Boxer RA, Gottesfeld I, Sharanjeet S, Lacorte MA, Parnell AV, Walker P. Noninvasive pulse oximetry in children with cyanotic congenital heart disease. Crit Care Med 1987, 15:1062-4.

3 Southall DP, Bignall S, Stebbens VA, Alexander JR, Rivers $R$, Lissauer T. Pulse oximeter and transcutaneous arterial oxygen measurements in neonatal and paediatric intensive care. Arch Dis Child 1987;62:882-8.

4 Fanconi S. Reliability of pulse oximetry in hypoxic infants. 7 Pediatr 1988;112:424 7

5 Severinghaus JW, Naifeh KH, Koh SO. Errors in 14 pulse oximeters during profound hypoxia. F Clin Monit 1989;5: oximet.

6 Abraham NG, Stebbens VA, Samuels MP, Southall DP Investigation of cyanotic/apnoeic episodes and sleep-related upper airway obstruction by long-term non-invasive bedside recordings. Pediatr Pulmonol 1990;8:259-62. 
7 Poets CF, Stebbens VA, Samuels MP, Southall DP. Patterns of breathing during hypoxaemia in preterm infants a around term (abstract). Early Hum Dev 1990;22:107-8.

8 Richards JM, Alexander JR, Shinebourne EA, de Swiet M Wilson AJ, Southall DP. Sequential 22-hour profiles of breathing patterns and heart rate in 110 full-term infants during their first 6 months of life. Pediatrics 1984:74: 763-77.

9 Anders T, Emde R, Parmelee A. A manual of standardized terminology techniques and criteria for scoring of states of sleep and wakefulness in newborm infants. Los Angeles: University of California (Los Angeles) Brain Information Service, 1971 .

10 Hoppenbrouwers T, Hodgman J, Arakawa K, Sterman MB Polysomnographic sleep and waking states are similar in
Paran subsequent siblings of SIDS and control infants during the first six months of life. Sleep 1989;12:265-76.

11 Coons S, Guilleminault C. Development of sleep-wake patterns and non-rapid eye movement sleep stages during the first six months of life in normal infants. Pediatrics 1982;69:793-8.

12 Flores-Guevara R, Sternberg B, Peirano P, Guidasci N, Durupt N, Monod N. Respiratory pauses and periodic breathing assessed by cardiopneumography in norma infants and in SIDS siblings. Neuropediatrics 1986;17: $59-62$.
13 Mok JYQ, McLaughlin FJ, Pintar M, Hak H, Amaro-Galvez $R$, Levison $H$. Transcutaneous monitoring of oxygenation: what is normal? $\mathcal{f}$ Pediatr 1986;108:365-71.

14 Stothers JK, Warner RM. Oxygen consumption and neonatal sleep states. F Physiol (Lond) 1978;278:435-40.

15 Henderson-Smart DJ, Read DJC. Reduced lung volume during behavioral active sleep in the newborn. $\mathcal{F} A p p l$ during behavioral active
Physiol 1979;46:1081-5.

16 Coleman JM, Mammel MC, Bing DR, Hagen EA, Boros SJ. Two vs. four channel pneumograms: the issue of $\mathrm{SaO}_{2}$ Two vs. four channel pneumograms:
monitoring. Pediatr Res 1989:25:305A.

17 Muttitt SC, Finer NN, Tierney AJ, Rossmann J. Neonatal apnea: diagnosis by nurse versus computer. Pediatrics 1988 ; 82:713-20.

18 Southall DP, Samuels MP, Talbert DG. Recurrent cyanotic episodes with severe arterial hypoxaemia and intrapulmonary shunting: a mechanism for sudden death. Arch Dis Child 1990;65:953-61.

19 Henderson-Smart DJ. Vulnerability to hypoxemia in the newborn. Sleep 1980;3:331-42.

20 Kelly DH, Shannon DC, O'Connell KC. Care of infants with near miss sudden infant death syndrome. Pediatrics 1978 ; 61:511-14.

21 Hunt CE, Brouillette RT, Hanson D. Theophylline improves pneumogram abnormalities in infants at risk for sudden pneumogram abnormalities in infants at risk for sudde
infant death syndrome. $\mathcal{F}$ Pediatr 1983;103:969-74. 